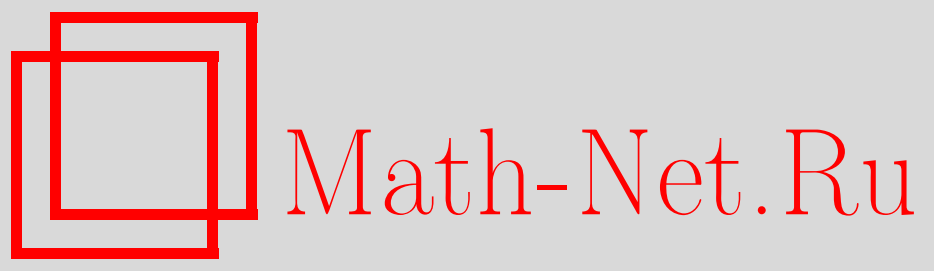

В. А. Авдеев, Стационарное распределение рейтинга игрока в модели Эло с одним соперником, Дискрет. матем., 2014, том 26, выпуск 4, 3-14

DOI: https://doi.org/10.4213/dm1299

Использование Общероссийского математического портала Math-Net.Ru подразумевает, что вы прочитали и согласны с пользовательским соглашением http: //www . mathnet.ru/rus/agreement

Параметры загрузки:

IP : 54.166 .219 .16

26 апреля 2023 г., 03:32:17

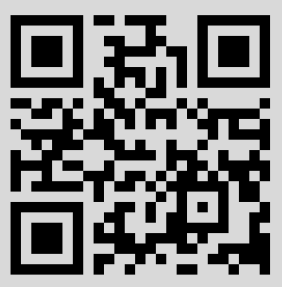




\title{
Стационарное распределение рейтинга игрока в модели Эло с одним соперником
}

\author{
() 2014 г. В. А. Авдеев*
}

\begin{abstract}
Рассматривается процесс изменения рейтинга игрока в бесконечной серии игр с одним и тем же соперником в модели Эло. Доказывается существование стационарного распределения этого процесса при некоторых предположениях о параметрах модели и находится его медиана в случае одинаковых уровней мастерства игроков.
\end{abstract}

Ключевые слова: модели рейтинговых систем, цепи Маркова, итерационные функциональные системы, предельные распределения.

\section{1. Введение}

Задача построения рейтинговой системы, позволяющей сравнивать всех игроков по результатам их отдельных игр друг с другом, исследуется уже очень давно.

Одной из первых статистически обоснованных моделей стала система Арпада Эло, принятая на вооружение Международной шахматной федерацией в 1970 году [3] и используемая в настоящее время не только в шахматах, но и в го, футболе и других видах спорта. Хотя с момента ее появления были предложены более общие рейтинговые системы, модель Эло в силу своей простоты при достаточной надежности результатов остается одной из самых распространенных рейтинговых систем.

Тем не менее, многие вопросы, касающиеся этой модели, до сих пор остаются предметом исследований. Один из них заключается в нахождении функции, наиболее адекватно описывающей совокупное распределение рейтингов всех игроков. Так, в работе [2] этот вопрос исследуется путем построения эволюционной стохастической модели и ее непрерывного приближения. В данной статье рассматривается вопрос о предельном распределении рейтинга в другом экстремальном случае, а именно при наличии всего лишь двух игроков. В таком случае модель представляет собой марковский процесс с дискретным временем и непрерывным пространством состояний.

Примеры похожих процессов возникают во многих других задачах, см., например, [6]. Исследование процессов такого рода тесно связано с теорией итерационных функциональных систем. Наиболее полные обзоры этой теории можно найти в работах [1] и [8]. Монотонным процессам посвящена также статья [4].

*Место работы: МГУ имени М. В.Ломоносова, e-mail: avdeev.vadim@gmail.com 


\section{2. Описание модели}

В модели Эло рейтинг игрока изменяется путем сравнения реального и прогнозируемого результатов матча. При построении прогноза предполагается, что в каждом матче побеждает более сильный игрок, но сила игрока в разных партиях может меняться под воздействием различных факторов, поэтому ее значения в разных партиях можно рассматривать как независимые одинаково распределенные случайные величины, среднее значение которых равняется уровню мастерства игрока. При этом под рейтингом подразумевается некоторая эмпирическая оценка мастерства.

При построении этой модели мы далее пользуемся следующими предположениями: во-первых, сила игрока имеет нормальное распределение, во-вторых, дисперсия этого распределения у всех игроков совпадает, и в-третьих, в играх не бывает ничьих.

Изменение рейтинга за одну партию описывается формулой

$$
R_{\text {new }}=R_{\text {old }}+k\left(W-W_{E}\right),
$$

где $k$ - константа, определяющая динамику изменения рейтинга, $W$ - реально набранное игроком число очков в партии (0 или 1) и $W_{E}$ - ожидаемое число набранных очков, которое равняется оценке вероятности победить соперника. Обозначая игроков буквами $A$ и $B$, будем записывать эти числа в виде $W^{A}, W_{E}^{A}$ и $W^{B}, W_{E}^{B}$ соответственно.

Обозначим также силу игроков через $p$ (от слова «рerformance») и уровень мастерства через $s$ (от слова «skill»).

Пусть сила первого игрока $p_{1}$ имеет нормальное распределение $\mathcal{N}\left(s_{1}, \sigma^{2}\right)$, а сила второго игрока, соответственно, $p_{2} \sim \mathcal{N}\left(s_{2}, \sigma^{2}\right)$. В таком случае разность $p_{1}-p_{2} \sim$ $\mathcal{N}\left(s_{1}-s_{2}, 2 \sigma^{2}\right)$. Первый игрок будет выигрывать тогда, когда случайная величина $p_{1}-p_{2}>0$. Доля таких матчей, то есть вероятность его победы, составит

$$
\mathbf{P}\left\{p_{1}-p_{2}>0\right\}=\Phi\left(\frac{s_{1}-s_{2}}{\sqrt{2} \sigma}\right),
$$

где $\Phi(x)$ - функция стандартного нормального распределения.

Так как уровни мастерства игроков являются скрытыми переменными и в реальности неизвестны, вместо них в расчетах используются сами рейтинги: по определению, $s_{i}=\mathbf{E} p_{i}$, a $R_{\text {old }}$ является оценкой математического ожидания $p_{i}$ по результатам предыдущих игр, поэтому в качестве оценки вероятности победы можно использовать $\Phi\left(\frac{R_{\text {old }}^{A}-R_{\mathrm{old}}^{B}}{\sqrt{2} \sigma}\right)$.

На практике используемые значения $k$ колеблются в пределах от 10 до 30 в зависимости от рейтинга игрока, а стандартное отклонение $\sigma=200$, но в нашей модели мы будем исследовать общий случай при условии $k \leqslant \sqrt{\pi} \sigma$.

Предположим, что два игрока $A$ и $B$ в некоторый момент имеют рейтинги $R_{0}^{A}$ и $R_{0}^{B}$ и уровни мастерства $s^{A}$ и $s^{B}$ соответственно, после чего начинают бесконечную серию игр только друг с другом. В таком случае рейтинг игрока $A$ меняется по следующему закону: $R_{\text {new }}^{A}=R_{\text {old }}^{A}+k\left(W^{A}-W_{E}^{A}\right)$, где

$$
\begin{aligned}
& W_{E}^{A}=\Phi\left(\frac{R_{\mathrm{old}}^{A}-R_{\mathrm{old}}^{B}}{\sqrt{2} \sigma}\right), \\
& W^{A}=\mathbf{1}_{p^{A}>p^{B}},
\end{aligned}
$$


то есть $W^{A}$ имеет распределение Бернулли $\mathcal{B}(1, q)$ с вероятностью успеха, равной $q=\Phi\left(\frac{s^{A}-s^{B}}{\sqrt{2} \sigma}\right)$.

Заметим, что $W^{A}+W^{B}=1$ и $W_{E}^{A}+W_{E}^{B}=1$, откуда

$$
R_{\text {new }}^{A}+R_{\text {new }}^{B}=R_{\text {old }}^{A}+R_{\text {old }}^{B},
$$

то есть сумма рейтингов игроков после каждой партии остается неизменной. Так как в начальный момент сумма рейтингов игроков равнялась $R_{0}^{A}+R_{0}^{B}$, то в каждый момент времени рейтинг игрока $B$ будет равен

$$
R^{B}=R_{0}^{A}+R_{0}^{B}-R^{A},
$$

откуда

$$
\frac{R_{\mathrm{old}}^{A}-R_{\mathrm{old}}^{B}}{\sqrt{2} \sigma}=\frac{2 R_{\mathrm{old}}^{A}-\left(R_{0}^{A}+R_{0}^{B}\right)}{\sqrt{2} \sigma} .
$$

Введем теперь следующие обозначения:

$$
\begin{aligned}
& \alpha=\frac{2}{\sqrt{2} \sigma}, \\
& \beta=-\frac{R_{0}^{A}+R_{0}^{B}}{\sqrt{2} \sigma} .
\end{aligned}
$$

Тогда общая модель изменения рейтинга игрока $A$ после $n$-й партии будет иметь следующий вид:

$$
R_{n}=R_{n-1}-k \Phi\left(\alpha R_{n-1}+\beta\right)+k W_{n},
$$

где все $W_{n}$ независимы и одинаково распределены, $W_{n} \sim \mathcal{B}(1, q)$ и $q, k, \alpha, \beta, R_{0}=R_{0}^{A}$ - константы, причем $0<q<1$ и $k, \alpha>0$.

\section{3. Итерационная система}

Определим итерационную функциональную систему $\mathcal{R}$. Для этого введем функции от $x \in \mathbb{R}$, соответствующие различным значениям случайной величины $W$ :

$$
\begin{aligned}
& f_{0}(x)=x-k \Phi(\alpha x+\beta), \\
& f_{1}(x)=x-k \Phi(\alpha x+\beta)+k,
\end{aligned}
$$

и сопоставим им вероятности $\{1-q, q\}$ соответственно. В дальнейшем будем под $f_{i}(x)$ понимать одну из функций $f_{0}(x)$ или $f_{1}(x)$ там, где это не имеет значения.

Отметим, что свойства функции нормального распределения далее используются только в лемме 1 , а для остальных доказательств важны только непрерывность $\Phi(x)$ и условие $0<\Phi(x)<1$.

Учитывая (2) и $(3)$, процесс $\left\{R_{n}(x)\right\}$ можно определить следующим образом:

$$
R_{n}(x)=f_{W_{n}}\left(R_{n-1}(x)\right)=\left(f_{W_{n}} \circ \ldots \circ f_{W_{1}}\right)(x) .
$$

Рассмотрим теперь вспомогательный процесс итераций аргумента, или, другими словами, процесс с обратным порядком композиции функций:

$$
\widetilde{R}_{n}(x)=\left(f_{W_{1}} \circ \ldots \circ f_{W_{n}}\right)(x) .
$$


Хотя для каждого $n$ распределения $R_{n}(x)$ и $\widetilde{R}_{n}(x)$ совпадают, процесс $\left\{\widetilde{R}_{n}(x)\right\}$, в отличие от $\left\{R_{n}(x)\right\}$, не является марковским и ведет себя абсолютно по-другому, что проиллюстрировано на рисунке 1 . В дальнейшем мы докажем, что процесс $\left\{\widetilde{R}_{n}(x)\right\}$ имеет поточечный предел, зависящий от конкретной реализации случайного процесса $\left\{W_{n}\right\}$.
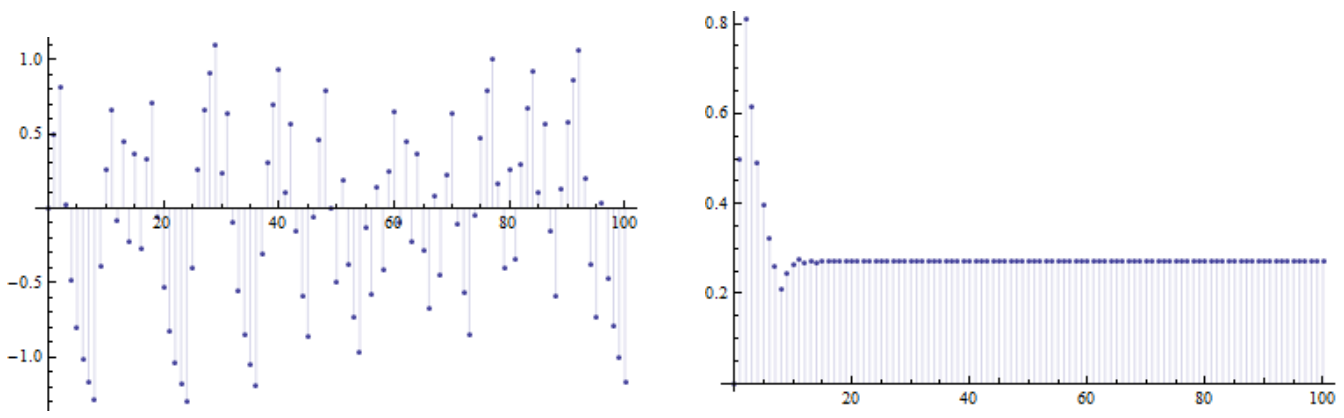

Рис. 1. Процессы $\left\{R_{n}\right\}$ и $\left\{\widetilde{R}_{n}\right\}$ при $q=\frac{1}{2}, k=\alpha=1, R_{0}=\beta=0, n \leqslant 100$.

В статье [5] доказывается теорема, связывающая распределения процессов с прямым и обратным порядком композиции непрерывных функций. Установим аналогичное утверждение для нашего случая.

Теорема 1. Пусть предел $\widetilde{R}_{\infty}(x)=\lim _{n \rightarrow+\infty} \widetilde{R}_{n}(x)$ существует почти наверное, конечен и не зависит от $x$. Тогда распределение случайной величины $\widetilde{R}_{\infty}$ является единственным стационарным распределением процесса $\left\{R_{n}(x)\right\}$.

Доказательство. Обозначим функции распределения случайных величин $R_{n}(x)$ и $\widetilde{R}_{n}(x)$ через $F_{n, x}(y)=\mathbf{P}\left\{R_{n}(x) \leqslant y\right\}$ и $\widetilde{F}_{n, x}(y)=\mathbf{P}\left\{\widetilde{R}_{n}(x) \leqslant y\right\}, y \in \mathbb{R}$.

Так как $\lim _{n \rightarrow+\infty} \widetilde{R}_{n}(x)=\widetilde{R}_{\infty}(x)$ почти наверное, то имеет место сходимость по распределению, то есть для всех непрерывных и ограниченных функций $h(y)$ выполняется равенство

$$
\lim _{n \rightarrow+\infty} \int_{\mathbb{R}} h(y) F_{n, x}(\mathrm{~d} y)=\int_{\mathbb{R}} h(y) \widetilde{F}_{\infty}(\mathrm{d} y) .
$$

С другой стороны, по определению $R_{n}(x)=f_{W_{n}}\left(R_{n-1}(x)\right)$, то есть с вероятностью $1-q$ это будет $f_{0}\left(R_{n-1}(x)\right)$ и с вероятностью $q$, соответственно, $f_{1}\left(R_{n-1}(x)\right)$. Следовательно,

$$
\int_{\mathbb{R}} h(y) F_{n, x}(\mathrm{~d} y)=\int_{\mathbb{R}}\left((1-q) h\left(f_{0}(y)\right)+q h\left(f_{1}(y)\right)\right) F_{n-1, x}(\mathrm{~d} y) .
$$

Заметим, что функции $f_{i}(y)$ непрерывны, а $h(x)$ непрерывна и ограничена, поэтому подынтегральная функция также является непрерывной и ограниченной.

При $n \rightarrow+\infty$ пределы $F_{n, x}(y)$ и $F_{n-1, x}(y)$ равняются $\widetilde{F}_{\infty}(y)$, поэтому

$$
\int_{\mathbb{R}} h(y) \widetilde{F}_{\infty}(\mathrm{d} y)=\int_{\mathbb{R}}\left((1-q) h\left(f_{0}(y)\right)+q h\left(f_{1}(y)\right)\right) \widetilde{F}_{\infty}(\mathrm{d} y) .
$$

Выберем теперь произвольное число $t$ и возьмем в качестве $h(y)$ последовательность непрерывных и ограниченных функций, имеющую в качестве предела функцию $\mathbf{1}_{(-\infty, t]}(y)$. 
Тогда в пределе в выражении (5) слева будет находиться функция распределения $\widetilde{F}_{\infty}(t)$, а справа она же после одного перехода процесса $\left\{R_{n}(x)\right\}$. Следовательно, $\widetilde{F}_{\infty}(y)$ является стационарным распределением процесса $R_{n}(x)$.

Так как утверждение теоремы верно для любого фиксированного начального состояния $x$, оно останется верным и в случае, когда начальное состояние - это случайная величина $X$.

Для доказательства единственности предположим теперь, что $X$ имеет некоторое стационарное распределение. Тогда и $R_{n}(X)$ будет иметь это стационарное распределение. Устремляя $n$ к бесконечности, получаем, что $\widetilde{R}_{\infty} \stackrel{d}{=} X$.

Заметим также, что из этого следует независимость стационарного распределения $R_{n}(X)$ от распределения $X$.

Дальнейшая наша цель состоит в доказательстве того, что для итерационной функциональной системы $\mathcal{R}$ выполняются условия теоремы 1 . Из формул (3) следует, что при больших положительных $x$ функции $f_{0}(x) \approx x-k$ и $f_{1}(x) \approx x$, так что при любом $q$ процесс будет смещаться влево по направлению к нулю. В свою очередь для больших по абсолютной величине отрицательных $x$ функции $f_{0}(x) \approx x$ и $f_{1}(x) \approx x+k$, так что процесс будет смещаться уже вправо.

Обозначим через $L(f)$ и $L_{x}(f)$ соответственно глобальную и локальную (в точке $x$ ) константы Липшица функции $f$ :

$$
\begin{aligned}
L(f) & =\sup _{x \neq y} \frac{|f(y)-f(x)|}{|y-x|}, \\
L_{x}(f) & =\limsup _{y \rightarrow x} \frac{|f(y)-f(x)|}{|y-x|}
\end{aligned}
$$

и найдем значения $L(f)$ для функций $f_{0}$ и $f_{1}$.

Лемма 1. При $k \leqslant \sqrt{\pi} \sigma$ справедливы неравенства: $0 \leqslant f_{i}^{\prime}(x)<1$, причем $L\left(f_{i}\right)=1$, $i=0,1$.

Доказательство. Заметим, что для всюду дифференцируемых функций $f(x)$ с ограниченной производной константы Липшица равны $L_{x}(f)=\left|f^{\prime}(x)\right|$ и $L(f)=$ $\sup _{x}\left|f^{\prime}(x)\right|$. Воспользовавшись этим, найдем значения $L_{x}(f)$ для функций $f_{0}$ и $f_{1}$ :

$$
L_{x}\left(f_{i}\right)=\left|f_{i}^{\prime}(x)\right|=\left|1-\frac{\alpha k}{\sqrt{2 \pi}} e^{-\frac{1}{2}(\alpha x+\beta)^{2}}\right| .
$$

В нашем случае $0<k \leqslant \sqrt{\pi} \sigma=\frac{\sqrt{2 \pi}}{\alpha}$, откуда $0<\frac{\alpha k}{\sqrt{2 \pi}} \leqslant 1$. Так как аргумент экспоненты $-\frac{1}{2}(\alpha x+\beta)^{2}$ не больше нуля, то аналогично $0<e^{-\frac{1}{2}(\alpha x+\beta)^{2}} \leqslant 1$, поэтому $0 \leqslant f_{i}^{\prime}(x)<1$ и знак абсолютной величины в (6) можно убрать. Наконец, при $x \rightarrow \pm \infty$ значение экспоненты в этой формуле стремится к нулю, поэтому $L\left(f_{i}\right)=$ $\sup _{x} L_{x}\left(f_{i}\right)=1$.

Так как глобальные константы Липшица функций $f_{0}$ и $f_{1}$ в нашем случае оказались равны единице, то к итерационной функциональной системе $\mathcal{R}$ нельзя применить теоремы о сильном сжатии [1]. Тем не менее, доказать выполнение необходимых нам условий можно методами статьи [7]. 


\section{4. Процесс $\widetilde{R}_{n}^{-1}(x)$}

Как следует из леммы 1 , в случае $k \leqslant \sqrt{\pi} \sigma$ выполняется неравенство $f_{i}^{\prime}(x) \geqslant 0$, причем $f_{i}^{\prime}(x)=0$ только при $k=\sqrt{\pi} \sigma$ и $x=-\frac{\beta}{\alpha}$, то есть не более, чем в одной точке. Таким образом, функции $f_{i}$ строго возрастают, и у них существуют обратные функции $f_{i}^{-1}$.

Возьмем теперь обратную функцию от определенной в (4) случайной функции $\widetilde{R}_{n}(x)$ и рассмотрим соответствующий марковский процесс:

$$
\widetilde{R}_{n}^{-1}(x)=\left(f_{W_{n}}^{-1} \circ \ldots \circ f_{W_{1}}^{-1}\right)(x),
$$

считая, что $\widetilde{R}_{0}^{-1}(x)=x$. Докажем сначала, что для любого $x$ предел $\widetilde{R}_{n}^{-1}(x)$ при $n \rightarrow+\infty$ равняется $\pm \infty$ почти наверное, а затем - что из этого факта следует необходимая нам независимость $\widetilde{R}_{\infty}(x)$ от $x$.

Введем следующее обозначение:

$$
g_{i}(x)=f_{i}^{-1}(x)-x
$$

где функциям $g_{0}$ и $g_{1}$ сопоставлены, как и функциям $f_{0}$ и $f_{1}$, вероятности $\{1-q, q\}$. Таким образом, можно определить случайную функцию $g(x)$, принимающую с вероятностью $1-q$ значение $g_{0}(x)$ и с вероятностью $q$ значение $g_{1}(x)$.

Лемма 2. Существует такое $T^{*} \in(0,+\infty)$, что

$$
\begin{gathered}
\mathbf{E} g\left(T^{*}\right)=(1-q) g_{0}\left(T^{*}\right)+q g_{1}\left(T^{*}\right)>0, \\
\mathbf{E} g\left(-T^{*}\right)=(1-q) g_{0}\left(-T^{*}\right)+q g_{1}\left(-T^{*}\right)<0 .
\end{gathered}
$$

Доказательство. Пусть $\varepsilon=\frac{1}{2} \min \{1-q, q\}$. Выберем число $T_{1}$ так, что $\Phi\left(\alpha T_{1}+\beta\right)>1-\varepsilon$. Тогда $-\Phi\left(\alpha T_{1}+\beta\right)+1<\varepsilon$ и

$$
f_{1}\left(T_{1}\right)=T_{1}-k \Phi\left(\alpha T_{1}+\beta\right)+k<T_{1}+k \varepsilon,
$$

откуда $T_{1}<f_{1}^{-1}\left(T_{1}+k \varepsilon\right)$ и $g_{1}\left(T_{1}+k \varepsilon\right)=f_{1}^{-1}\left(T_{1}+k \varepsilon\right)-\left(T_{1}+k \varepsilon\right)>-k \varepsilon$. Заметим, что функция $\Phi(x)$ строго возрастающая, поэтому

$$
\Phi\left(\alpha\left(T_{1}+k\right)+\beta\right)>\Phi\left(\alpha T_{1}+\beta\right)>1-\varepsilon,
$$

а значит, верна аналогичная оценка:

$$
f_{0}\left(T_{1}+k\right)=T_{1}+k-k \Phi\left(\alpha\left(T_{1}+k\right)+\beta\right)<T_{1}+k \varepsilon,
$$

откуда $T_{1}+k<f_{0}^{-1}\left(T_{1}+k \varepsilon\right)$ и $g_{0}\left(T_{1}+k \varepsilon\right)=f_{0}^{-1}\left(T_{1}+k \varepsilon\right)-\left(T_{1}+k \varepsilon\right)>k-k \varepsilon$. Следовательно,

$$
\begin{aligned}
\mathbf{E} g\left(T_{1}+k \varepsilon\right)=(1-q) g_{0} & \left(T_{1}+k \varepsilon\right)+q g_{1}\left(T_{1}+k \varepsilon\right)> \\
& >(1-q)(k-k \varepsilon)-q k \varepsilon=k(1-q-\varepsilon) .
\end{aligned}
$$

Так как $\varepsilon$ был выбран таким, что $\varepsilon<1-q$, то $\mathbf{E} g\left(T_{1}+k \varepsilon\right)>0$.

Аналогично выбирая $T_{0}$ так, что $\Phi\left(\alpha T_{0}+\beta\right)<\varepsilon$, получаем, что

$$
f_{0}\left(T_{0}\right)=T_{0}-k \Phi\left(\alpha T_{0}+\beta\right)>T_{0}-k \varepsilon
$$


откуда $T_{0}>f_{0}^{-1}\left(T_{0}-k \varepsilon\right)$ и $g_{0}\left(T_{0}-k \varepsilon\right)=f_{0}^{-1}\left(T_{0}-k \varepsilon\right)-\left(T_{0}-k \varepsilon\right)<k \varepsilon$. Из строгого возрастания функции $\Phi(x)$ следует, что

$$
\Phi\left(\alpha\left(T_{0}-k\right)+\beta\right)<\Phi\left(\alpha T_{0}+\beta\right)<\varepsilon,
$$

поэтому

$$
f_{1}\left(T_{0}-k\right)=T_{0}-k-k \Phi\left(\alpha\left(T_{0}-k\right)+\beta\right)+k>T_{0}-k \varepsilon,
$$

а значит, $T_{0}-k>f_{1}^{-1}\left(T_{0}-k \varepsilon\right)$ и $g_{1}\left(T_{0}-k \varepsilon\right)=f_{1}^{-1}\left(T_{0}-k \varepsilon\right)-\left(T_{0}-k \varepsilon\right)<k \varepsilon-k$. Отсюда

$$
\begin{aligned}
\mathbf{E} g\left(T_{0}-k \varepsilon\right)=(1-q) g_{0} & \left(T_{0}-k \varepsilon\right)+q g_{1}\left(T_{0}-k \varepsilon\right)< \\
& <(1-q) k \varepsilon+q(k \varepsilon-k)=k(\varepsilon-q) .
\end{aligned}
$$

Пользуясь тем, что $\varepsilon<q$, получаем, что $\mathbf{E} g\left(T_{0}-k \varepsilon\right)<0$.

Полагая $T^{*}=\max \left\{\left|T_{0}-k \varepsilon\right|,\left|T_{1}+k \varepsilon\right|\right\}$, получаем окончательно, что $\mathbf{E} g\left(T^{*}\right)>0$ и $\mathbf{E} g\left(-T^{*}\right)<0$.

Заметим теперь, что по определению

$$
g_{W_{n}}\left(\widetilde{R}_{n-1}^{-1}(x)\right)=f_{W_{n}}^{-1}\left(\widetilde{R}_{n-1}^{-1}(x)\right)-\widetilde{R}_{n-1}^{-1}(x)=\widetilde{R}_{n}^{-1}(x)-\widetilde{R}_{n-1}^{-1}(x),
$$

то есть $g_{W_{n}}\left(\widetilde{R}_{n-1}^{-1}(x)\right)$ - это смещение процесса $\left\{\widetilde{R}^{-1}(x)\right\}$ на $n$-м шаге. Более того, из неравенства

$$
f_{0}(x)=x-k \Phi(\alpha x+\beta)<x
$$

следует, что $g_{0}(x)=f_{0}^{-1}(x)-x>0$, и аналогично из неравенства

$$
f_{1}(x)=x+k(1-\Phi(\alpha x+\beta))>x
$$

следует, что $g_{1}(x)=f_{1}^{-1}(x)-x<0$. Другими словами, смещение процесса $\left\{\widetilde{R}_{n}^{-1}\right\}$ из точки $x$ направо равняется $g_{0}(x)$, а налево $-g_{1}(x)$.

Кроме того, по лемме 1 функции $f_{i}$ таковы, что $0 \leqslant f_{i}^{\prime}(x)<1$, откуда

$$
\left(f_{i}^{-1}\right)^{\prime}(x)=\frac{1}{f_{i}^{\prime}\left(f_{i}^{-1}(x)\right)}>1 .
$$

Следовательно, $g_{i}^{\prime}(x)=\left(f_{i}^{-1}(x)\right)^{\prime}-1>0$, то есть функции $g_{i}$ возрастают.

Докажем теперь следующую лемму:

Лемма 3. Для любых действительного $x$ и положительного $T$ процесс $\left\{\widetilde{R}_{n}^{-1}(x)\right\}$ выходит за предель отрезка $[-T, T]$ почти наверное.

Доказательство. Положим

$$
\varepsilon^{+}(x)=\sup \{\varepsilon \mid \mathbf{P}\{g(x) \geqslant \varepsilon\} \geqslant \varepsilon\} .
$$

Таким образом, из любой точки $x$ происходит смещение процесса вправо не менее, чем на $\varepsilon^{+}(x)$, с вероятностью не менее, чем $\varepsilon^{+}(x)$. Так как вероятность смещения процесса вправо всегда равняется $1-q$, то данная величина равняется минимуму из соответствующих расстояния и вероятности: $\varepsilon^{+}(x)=\min \left\{g_{0}(x), 1-q\right\}$. Аналогично положим

$$
\varepsilon^{-}(x)=\sup \{\varepsilon \mid \mathbf{P}\{g(x) \leqslant-\varepsilon\} \geqslant \varepsilon\},
$$


тогда из любой точки $x$ происходит смещение влево не менее, чем на $\varepsilon^{-}(x)$, с вероятностью не менее, чем $\varepsilon^{-}(x)$. Так как вероятность смещения процесса влево всегда равняется $q$, то $\varepsilon^{-}(x)=\min \left\{-g_{1}(x), q\right\}$.

Так как вероятности $q, 1-q>0$ и $g_{0}(x)>0, g_{1}(x)<0$, то обе функции $\varepsilon^{+}(x)$ и $\varepsilon^{-}(x)$ положительны. Поэтому из компактности отрезка $[-T, T]$ и непрерывности функций $\varepsilon^{+}(x), \varepsilon^{-}(x)$ следует, что $\varepsilon^{+}=\inf _{x \in[-T, T]} \varepsilon^{+}(x)$ и $\varepsilon^{-}=\inf _{x \in[-T, T]} \varepsilon^{-}(x)$ также больше нуля.

Заметим, что по условию достаточно рассмотреть процесс $\left\{\widetilde{R}_{n}^{-1}\left(x_{0}\right)\right\}$ при $\left|x_{0}\right| \leqslant T$. Для того, чтобы дойти из точки $x_{0}$ до правого конца отрезка $[-T, T]$, заведомо хватит $N^{+}=\left[\frac{2 T}{\varepsilon^{+}}\right]+1$ шагов величины $\varepsilon^{+}$вправо. Так как вероятность каждого такого шага не меньше $\varepsilon^{+}$, то вероятность, исходя из $x_{0}$, выйти за правую границу отрезка за $N^{+}$шагов не меньше $\left(\varepsilon^{+}\right)^{N^{+}}$.

Аналогично дойти из точки $x_{0}$ до левого конца отрезка $[-T, T]$ можно за $N^{-}=$ $\left[\frac{2 T}{\varepsilon^{-}}\right]+1$ шагов величины $\varepsilon^{-}$влево. Вероятность каждого такого шага не меньше $\varepsilon^{-}$, поэтому вероятность, исходя из $x_{0}$, выйти за левую границу отрезка за $N^{-}$шагов не меньше $\left(\varepsilon^{-}\right)^{N^{-}}$.

Учитывая, что $\varepsilon^{+}, \varepsilon^{-}>0$, получаем, что вероятность $\tau\left(x_{0}\right)$ остаться в пределах отрезка $[-T, T]$ за $N^{*}=\max \left\{N^{-}, N^{+}\right\}$шагов не больше $1-\left(\varepsilon^{+}\right)^{N^{+}}-\left(\varepsilon^{-}\right)^{N^{-}}$. Следовательно,

$$
\tau=\sup _{x_{0} \in[-T, T]} \tau\left(x_{0}\right) \leqslant 1-\left(\varepsilon^{+}\right)^{N^{+}}-\left(\varepsilon^{-}\right)^{N^{-}}<1 .
$$

Если за $N^{*}$ шагов марковский процесс $\widetilde{R}_{n}^{-1}\left(x_{0}\right)$ не вышел за пределы отрезка $[-T, T]$, то рассуждения можно повторить с новым значением $x_{0}$, равным $\widetilde{R}_{N^{*}}^{-1}\left(x_{0}\right)$. Поэтому вероятность остаться в пределах отрезка за $N$ таких итераций по $N^{*}$ шагов не превосходит $\tau^{N}$ и стремится к нулю при $N \rightarrow+\infty$.

Следовательно, для любого $x_{0}$ процесс $\widetilde{R}_{n}^{-1}\left(x_{0}\right)$ выходит за пределы отрезка $[-T, T]$ почти наверное.

Следующий шаг состоит в доказательстве того, что $\widetilde{R}_{n}^{-1}(x)$ уходит на бесконечность почти наверное.

Лемма 4. Для любого $x$ предел $\lim _{n \rightarrow+\infty} \widetilde{R}_{n}^{-1}(x)$ равен $+\infty$ или $-\infty$ почти наверное.

Доказательство. Предположим без ограничения общности, что первый выход процесса $\widetilde{R}_{n}^{-1}(x)$ за пределы отрезка $\left[-T^{*}, T^{*}\right]$, где $T^{*}$ - выбранное в лемме 2 число, был вправо. Обозначим этот момент через $N_{0}=\min \left\{n \mid \widetilde{R}_{n}^{-1}(x)>T^{*}\right\}$ и рассмотрим процесс $\left\{\widetilde{R}^{-1}(x)\right\}$ в момент $N_{0}+n$. Так как $g_{W_{n}}\left(\widetilde{R}_{n-1}^{-1}(x)\right)$ - это смещение процесса $\left\{\widetilde{R}^{-1}(x)\right\}$ на $n$-м шаге, то состояние процесса в момент $N_{0}+n$ можно представить следующим образом:

$$
\widetilde{R}_{N_{0}+n}^{-1}(x)=\widetilde{R}_{N_{0}}^{-1}(x)+\sum_{i=1}^{n} g_{W_{N_{0}+i}}\left(\widetilde{R}_{N_{0}+i-1}^{-1}(x)\right) .
$$

Рассмотрим сумму

$$
G_{n}=\sum_{i=1}^{n} g_{W_{N_{0}+i}}\left(T^{*}\right)
$$


Как уже было доказано в лемме $2, \mathbf{E} g_{W_{N_{0}+i}}\left(T^{*}\right)>0$, причем все функции $g_{W_{N_{0}+i}}$ независимы и одинаково распределены, поэтому по закону больших чисел предел их суммы $\lim _{n \rightarrow+\infty} G_{n}$ равен $+\infty$ почти наверное. Как следствие, случайное событие $H_{+}$, состоящее в том, что $G_{l}=\sum_{i=1}^{l} g_{W_{N_{0}+i}}\left(T^{*}\right)>0$ для любого $l$, имеет положительную вероятность $\eta_{+}$, не зависящую от $x$.

Докажем по индукции, что если выполняется событие $H_{+}$, то $\widetilde{R}_{N_{0}+j}^{-1}(x)>T^{*}$ для всех $j \geqslant 0$. База индукции, то есть случай $j=0$, следует из того, что за $N_{0}$ шагов процесс $\left\{\widetilde{R}^{-1}(x)\right\}$ вышел за пределы отрезка $\left[-T^{*}, T^{*}\right]$ вправо. Пусть утверждение выполнено для всех $j \leqslant J-1$, докажем его для $j=J$ :

$$
\widetilde{R}_{N_{0}+J}^{-1}(x)=\widetilde{R}_{N_{0}}^{-1}(x)+\sum_{i=1}^{J} g_{W_{N_{0}+i}}\left(\widetilde{R}_{N_{0}+i-1}^{-1}(x)\right)>T^{*}+\sum_{i=1}^{J} g_{W_{N_{0}+i}}\left(T^{*}\right)
$$

в силу индуктивного предположения и того, что функции $g_{W_{N_{0}+i}}$ возрастают. В свою очередь, из выполнения события $H_{+}$следует, что $T^{*}+\sum_{i=1}^{J} g_{W_{N_{0}+i}}\left(T^{*}\right)>T^{*}$, что и требовалось.

Так как $\widetilde{R}_{N_{0}+j}^{-1}(x)>T^{*}$, то состояние процесса в момент $N_{0}+n$ можно оценить следующим образом:

$$
\begin{aligned}
\widetilde{R}_{N_{0}+n}^{-1}(x)=\widetilde{R}_{N_{0}}^{-1}(x)+\sum_{i=1}^{n} g_{W_{N_{0}+i}} & \left(\widetilde{R}_{N_{0}+i-1}^{-1}(x)\right)> \\
& >T^{*}+\sum_{i=1}^{n} g_{W_{N_{0}+i}}\left(T^{*}\right)=T^{*}+G_{n} .
\end{aligned}
$$

Так как $G_{n} \rightarrow+\infty$ почти наверное, то при выполнении события $H_{+}$процесс $\widetilde{R}_{N_{0}+n}^{-1}(x) \rightarrow+\infty$ почти наверное.

Аналогично рассматривая симметричный случай $\widetilde{R}_{N_{0}}^{-1}(x)<-T^{*}$ и событие $H_{-}$, имеющее вероятность $\eta_{-}>0$, получаем, что при выполнении этого события процесс $\widetilde{R}_{N_{0}+n}^{-1}(x) \rightarrow-\infty$ почти наверное.

Таким образом, после того, как процесс $\widetilde{R}_{n}^{-1}(x)$ вышел за пределы отрезка $\left[-T^{*}, T^{*}\right]$, он с некоторой положительной вероятностью $\eta$ больше в него не вернется и уйдет либо в $+\infty$, либо в $-\infty$.

Если после первого выхода за границы отрезка $\left[-T^{*}, T^{*}\right]$ марковский процесс $\widetilde{R}_{n}^{-1}(x)$ вернулся в него обратно, то рассуждения можно повторить с новым значением $x$, взяв за него точку возвращения процесса. Поэтому вероятность вернуться в отрезок в $N$-й раз не превосходит $(1-\eta)^{N}$ и стремится к нулю при $N \rightarrow+\infty$.

Следовательно, для любого $x$ предел $\lim _{n \rightarrow+\infty} \widetilde{R}_{n}^{-1}(x)$ почти наверное равен $\pm \infty$.

На основе леммы 4 теперь несложно доказать выполнение одного из необходимых условий теоремы 1 , а именно, что предел $\lim _{n \rightarrow+\infty} \widetilde{R}_{n}(x)$ существует почти наверное и не зависит от $x$.

Лемма 5. Случайная величина $\lim _{n \rightarrow+\infty} \widetilde{R}_{n}(x)$ существует почти наверное и не зависит от $x$.

Доказательство. Так как функции $g_{i}(x)$ возрастают, то и $f_{i}^{-1}(x)=g_{i}(x)+x$ также возрастают, а значит, возрастают и функции $\widetilde{R}_{n}^{-1}(x)$ как их композиции.

Пусть $Y$ - счетное всюду плотное в $\mathbb{R}$ множество. Тогда, используя лемму 4 и не рассматривая множество нулевой меры, можно считать, что $\lim _{n \rightarrow+\infty} \widetilde{R}_{n}^{-1}(y)= \pm \infty$ 
для любого $y \in Y$. Заметим, что если этот предел равен $+\infty$, то он равен $+\infty$ и для всех чисел больших $y$, в силу возрастания $\widetilde{R}_{n}^{-1}(y)$. Аналогично, если он равен $-\infty$, то такой же предел будет и у всех чисел, меньших $y$.

Рассмотрим теперь случайную величину

$$
y_{0}=\inf _{y \in Y}\left\{\lim _{n \rightarrow+\infty} \widetilde{R}_{n}^{-1}(y)=+\infty\right\}=\sup _{y \in Y}\left\{\lim _{n \rightarrow+\infty} \widetilde{R}_{n}^{-1}(y)=-\infty\right\} .
$$

Для любого $\varepsilon>0$ существуют такие случайные величины $y_{+}, y_{-}$, принимающие значения в $Y$, что выполняется одно из следующих условий. Если $y_{0}$ конечен, то $y_{-}<y_{0}<y_{+}$, причем $y_{+}-y_{-}<\varepsilon$. Если $y_{0}=+\infty$, то $\frac{1}{\varepsilon}<y_{-}=y_{+}$. Наконец, если $y_{0}=-\infty$, то $y_{-}=y_{+}<-\frac{1}{\varepsilon}$.

Выберем теперь произвольное число $x \in \mathbb{R}$. Для достаточно большого $N$, зависящего от $x$ и реализации случайного процесса $\left\{W_{n}\right\}$, будет выполняться одна из следующих возможностей. Если $y_{0}$ конечен, то $\widetilde{R}_{N}^{-1}\left(y_{-}\right)<x$ и $\widetilde{R}_{N}^{-1}\left(y_{+}\right)>x$, откуда $y_{-}<\widetilde{R}_{N}(x)<y_{+}$, причем $y_{+}-y_{-}<\varepsilon$. Если $y_{0}=+\infty$, то $\widetilde{R}_{N}^{-1}\left(y_{-}\right)<x$ и $\frac{1}{\varepsilon}<y_{-}$, откуда $\widetilde{R}_{N}(x)>y_{-}>\frac{1}{\varepsilon}$. Наконец, если $y_{0}=-\infty$, то $\widetilde{R}_{N}^{-1}\left(y_{+}\right)>x$ и $y_{+}<-\frac{1}{\varepsilon}$, откуда $\widetilde{R}_{N}(x)<y_{+}<-\frac{1}{\varepsilon}$.

Так как эти утверждения верны для любого $\varepsilon>0$, во всех трех случаях получаем, что

$$
\lim _{n \rightarrow+\infty} \widetilde{R}_{n}(x)=y_{0}
$$

то есть рассматриваемый предел существует почти наверное и не зависит от $x$.

Таким образом, мы доказали, что предел $\lim _{n \rightarrow+\infty} \widetilde{R}_{n}(x)$ существует почти наверное и не зависит от $x$. Для доказательства выполнения условий теоремы 1 осталось убедиться, что он к тому же конечен.

Лемма 6. $\lim _{n \rightarrow+\infty} \widetilde{R}_{n}(x)$ конечен почти наверное.

Доказательство. Пусть $\widetilde{G}_{n}=\sum_{i=1}^{n} g_{W_{i}}\left(T^{*}\right)$, при этом $\widetilde{G}_{0}=0$. Рассмотрим событие $\left\{\min _{n \geqslant 0} \widetilde{G}_{n}>-t\right\}$. Докажем по индукции, что если это событие выполняется, TO

$$
\widetilde{R}_{n}^{-1}\left(T^{*}+t\right) \geqslant T^{*}+t+\widetilde{G}_{n} .
$$

База индукции, то есть случай $n=0$, следует из того, что по определению $\widetilde{R}_{0}^{-1}\left(T^{*}+t\right)=T^{*}+t$ и $\widetilde{G}_{0}=0$. Пусть утверждение выполнено для всех $n \leqslant N-1$, докажем его для $n=N$ :

$$
\begin{aligned}
\widetilde{R}_{N}^{-1}\left(T^{*}+t\right)=\widetilde{R}_{N-1}^{-1}\left(T^{*}+t\right) & +g_{W_{N}}\left(\widetilde{R}_{N-1}^{-1}\left(T^{*}+t\right)\right) \geqslant \\
& \geqslant T^{*}+t+\widetilde{G}_{N-1}+g_{W_{N}}\left(T^{*}+t+\widetilde{G}_{N-1}\right)
\end{aligned}
$$

в силу предположения индукции и того, что функция $g_{W_{N}}$ возрастает. Снова пользуясь возрастанием этой функции и тем, что $\min _{n \geqslant 0} \widetilde{G}_{n}>-t$, последнее выражение можно оценить снизу следующим образом:

$$
\begin{aligned}
T^{*}+t+ & \widetilde{G}_{N-1}+g_{W_{N}}\left(T^{*}+t+\widetilde{G}_{N-1}\right) \geqslant \\
& \geqslant T^{*}+t+\widetilde{G}_{N-1}+g_{W_{N}}\left(T^{*}+t-t\right)=T^{*}+t+\widetilde{G}_{N},
\end{aligned}
$$

что и требовалось. Таким образом, $\widetilde{R}_{n}^{-1}\left(T^{*}+t\right) \geqslant T^{*}+t+\widetilde{G}_{n}>T^{*}$, откуда $\lim _{n \rightarrow+\infty} \widetilde{R}_{n}^{-1}\left(T^{*}+t\right) \geqslant T^{*}$. 
Аналогично пределу $G_{n}, \lim _{n \rightarrow+\infty} \widetilde{G}_{n}=+\infty$ почти наверное, поэтому

$$
\lim _{t \rightarrow+\infty} \mathbf{P}\left\{\min _{n \geqslant 0} \widetilde{G}_{n}>-t\right\}=1 .
$$

Следовательно,

$$
\lim _{t \rightarrow+\infty} \mathbf{P}\left\{\lim _{n \rightarrow+\infty} \widetilde{R}_{n}^{-1}\left(T^{*}+t\right) \geqslant T^{*}\right\}=1,
$$

откуда

$$
\lim _{t \rightarrow+\infty} \mathbf{P}\left\{\lim _{n \rightarrow+\infty} \widetilde{R}_{n}^{-1}(t)=-\infty\right\}=0 .
$$

По определению $y_{0}$ это означает, что $\mathbf{P}\left\{y_{0}=+\infty\right\}=0$. Отсюда и из доказанного в лемме 5 соотношения (7) следует, что

$$
\mathbf{P}\left\{\lim _{n \rightarrow+\infty} \widetilde{R}_{n}(x)=+\infty\right\}=0
$$

для любого $x \in \mathbb{R}$. Аналогично рассматривая симметричный случай и сумму $\sum_{i=1}^{n} g_{W_{i}}\left(-T^{*}\right)$, получаем, что $\mathbf{P}\left\{\lim _{n \rightarrow+\infty} \widetilde{R}_{n}(x)=-\infty\right\}=0$ для любого $x \in \mathbb{R}$.

Следовательно, предел $\widetilde{R}_{n}(x)$ при $n \rightarrow+\infty$ конечен почти наверное.

Таким образом, мы окончательно получаем следующую теорему:

Теорема 2. Процесс $\left\{R_{n}\right\}$ имеет единственное стационарное распределение.

Доказательство. По лемме 5 предел $\widetilde{R}_{\infty}(x)=\lim _{n \rightarrow+\infty} \widetilde{R}_{n}(x)$ существует почти наверное и не зависит от $x$, а по лемме 6 он к тому же конечен.

Следовательно, по теореме 1 распределение $\widetilde{R}_{\infty}$ является единственным стационарным распределением процесса $\left\{R_{n}(x)\right\}$, что и требовалось.

В заключение рассмотрим случай одинаковых уровней мастерства у игрока и его соперника и найдем медиану стационарного распределения рейтинга игрока при этом условии.

Теорема 3. При $s_{A}=s_{B}$ стационарное распределение симметрично и его медиана $m=\frac{R_{0}^{A}+R_{0}^{B}}{2}=-\frac{\beta}{\alpha}$.

Доказательство. Так как $s_{A}=s_{B}$, то $q=\Phi\left(\frac{s^{A}-s^{B}}{\sqrt{2} \sigma}\right)=\Phi(0)=\frac{1}{2}$, а значит, случайная величина $W$ имеет стандартное распределение Бернулли и $W \sim 1-W$.

Заметим теперь, что выражение (2) для изменения рейтинга игрока в таком случае полностью совпадает с выражением для изменения рейтинга его соперника. Так как по теореме 1 стационарное распределение для такого процесса не зависит от его начального распределения, то стационарные распределения рейтингов игрока и его соперника совпадают.

Следовательно, из условия (1) сохранения суммы рейтингов получаем, что

$$
\mathbf{P}\left\{R_{\infty}^{A} \leqslant x\right\}=\mathbf{P}\left\{R_{\infty}^{B} \leqslant x\right\}=\mathbf{P}\left\{R_{0}^{A}+R_{0}^{B}-R_{\infty}^{A} \leqslant x\right\} .
$$

Отсюда

$$
\mathbf{P}\left\{R_{\infty}^{A} \leqslant m+x\right\}=\mathbf{P}\left\{R_{\infty}^{A} \geqslant m-x\right\},
$$

где $m=\frac{R_{0}^{A}+R_{0}^{B}}{2}$, то есть стационарное распределение является симметричным с медианой $m$.

Наконец, равенство $\frac{R_{0}^{A}+R_{0}^{B}}{2}=-\frac{\beta}{\alpha}$ следует из определения $\alpha$ и $\beta$. 


\section{Список литературы}

1. P. Diaconis, D. Freedman, "Iterated random functions", SIAM Review, 41:1 (1999), 45-76.

2. T. Fenner, M. Levene, G. Loizou, "A discrete evolutionary model for chess players' ratings", IEEE Trans. Comput. Intell. AI in Games, 4:2 (2012), 84-93.

3. M. E. Glickman, "A comprehensive guide to chess ratings", Amer. Chess J., 3 (1995), 59-102.

4. T. Kamihigashi, J. Stachurski, "Asymptotics of stochastic recursive economies under monotonicity", KIER Working Papers, 666 (2009).

5. G. Letac, "A contraction principle for certain Markov chains and its applications", Random matrices and their applications, Contemp. Math., 50, Amer. Math. Soc., 1986, 263-273.

6. D. Steinsaltz, "Zeno's walk: A random walk with refinements", Probab. Theory Related Fields, 107:1 (1997), 99-121.

7. D. Steinsaltz, "Locally contractive iterated function systems", Ann. Prob., 27:4 (1999), 1952-1979.

8. Ö. Stenflo, "A survey of average contractive iterated function systems", J. Differ. Equations Appl., 18:8 (2012), 1355-1380. 